\title{
BMJ Open Vascular and cognitive effects of cocoa- rich chocolate in postmenopausal women: a study protocol for a randomised clinical trial
}

\author{
Irene A Garcia-Yu, ${ }^{1,2}$ Luis Garcia-Ortiz, ${ }^{1,3}$ Manuel A Gómez-Marcos, , ${ }^{1,4}$ \\ Rosario Alonso-Dominguez, ${ }^{1}$ Jesus Gonzalez-Sanchez, ${ }^{1,5}$ Sara Mora-Simon, ${ }^{1,6}$ \\ Susana González-Manzano, ${ }^{7}$ Emiliano Rodriguez-Sanchez, ${ }^{1,4}$ \\ Jose A Maderuelo-Fernandez, ${ }^{1}$ Jose I Recio-Rodriguez ${ }^{1,8}$
}

To cite: Garcia-Yu IA, GarciaOrtiz L, Gómez-Marcos MA, et al. Vascular and cognitive effects of cocoa-rich chocolate in postmenopausal women: a study protocol for a randomised clinical trial. BMJ Open 2018;8:e24095. doi:10.1136/ bmjopen-2018-024095

- Prepublication history for this paper is available online. To view these files, please visit the journal online (http://dx.doi. org/10.1136/bmjopen-2018024095).

JAM-F and JIR-R contributed equally.

Received 9 May 2018

Revised 3 October 2018

Accepted 2 November 2018

Check for updates

(c) Author(s) (or their employer(s)) 2018. Re-use permitted under CC BY-NC. No commercial re-use. See rights and permissions. Published by BMJ.

For numbered affiliations see end of article.

Correspondence to

Irene A Garcia-Yu;

ireneailinggarciayu@gmail.com

\section{ABSTRACT}

Introduction The intake of polyphenols has certain health benefits. This study will aim to assess the effect of adding a daily amount of chocolate high in cocoa content and polyphenols to the normal diet on blood pressure, vascular function, cognitive performance, quality of life and body composition in postmenopausal women.

Methods and analysis Here we plan a randomised clinical trial with two parallel groups involving a total of 140 women between 50 and 64 years in the postmenopausal period, defined by amenorrhoea of at least 12 consecutive months. The main variable will be the change in blood pressure. Secondary variables will be changes in vascular function, quality of life, cognitive performance and body composition. The intervention group will be given chocolate containing $99 \%$ cocoa, with instructions to add $10 \mathrm{~g}$ daily to their normal diet for 6 months. The daily nutritional contribution of this amount of chocolate is $59 \mathrm{kcal}$ and $65.4 \mathrm{mg}$ of polyphenols. There will be no intervention in the control group. All variables will be measured at the baseline visit and 3 and 6 months after randomisation, except cognitive performance and quality of life, which will only be assessed at baseline and at 6 months. Recruitment is scheduled to begin on 1 June 2018, and the study will continue until 31 May 2019.

Ethics and dissemination This study was approved by the Clinical Research Ethics Committee of the Health Area of Salamanca, Spain ('CREC of Health Area of Salamanca'), in February 2018. A SPIRIT checklist is available for this protocol. The clinical trial has been registered at ClinicalTrials.gov provided by the US National Library of Medicine, number NCT03492983. The results will be disseminated through open access peer-reviewed journals, conference presentations, broadcast media and a presentation to stakeholders.

\section{INTRODUCTION}

Polyphenols are bioactive compounds found in many plants, fruits and vegetables. The beneficial effects on human health associated with the consumption of a diet rich in polyphenols have generated great scientific

\section{Strengths and limitations of this study}

- This study will use commercially available chocolate with a high content of cocoa and polyphenols during the intervention.

- Blood pressure and vascular function will be measured objectively using a sphygmomanometer and a Vasera VS-2000 device (Fukuda Denshi), with body composition measured by impedance analysis, while the quality of life and cognitive performance will be assessed using validated instruments.

- Due to the nature of the intervention, the participants cannot be blinded, although the researchers who perform the measurements and the statistical analysis will be blinded.

interest in these substances. ${ }^{1-3}$ The action of polyphenols is based on their antioxidant capacity through the uptake of free radicals, the chelation of metals with redox properties and the modulation and inhibition of enzymatic activities. ${ }^{4}$

The most abundant polyphenols in cocoa are flavonoids, which have been linked to a protective effect against cardiovascular disease, decreasing the risk of cardiovascular morbidity and mortality and favouring the prevention of other chronic diseases, such as diabetes mellitus type $2 .{ }^{1-3} 5-7$ The ability to reduce cardiovascular risk could be due to an improvement in the elements that define metabolic syndrome, the improvement of vascular endothelial dysfunction, insulin resistance and the inhibition of platelet activation and aggregation. ${ }^{8}$ However, although current evidence suggests that polyphenols produce an improvement in cardiovascular health, this is insufficient to determine the minimum amount of intake necessary to achieve health benefits. ${ }^{10}$ 


\section{Cocoa polyphenols and blood pressure}

The effect of consuming polyphenols present in chocolate on the blood pressure statistics of healthy individuals is unclear. Cocoa consumption has been associated with an improvement in endothelial function and a decrease in blood pressure in both healthy subjects and those with risk factors and cardiovascular diseases. ${ }^{11} 12$ Some studies have observed a dose-dependent relationship between cocoa intake and clinical blood pressure, with higher consumption equated to lower blood pressure and better vascular function. ${ }^{13}$ Conversely, other research has not obtained significant changes in these parameters related to the supplementation of cocoa or pure polyphenols, such as epicatechin or quercetin. ${ }^{1516}$

Endothelial dysfunction in postmenopausal women causes changes that favour the development of cardiovascular risk factors and atherosclerosis, which lead to the appearance and maintenance of hypertension. ${ }^{17} 18$ A decrease in blood pressure has been observed in this group after daily consumption of cocoa with a flavonol content of $40.12 \mathrm{mg}$. Below this level, however, no changes have been observed. ${ }^{19}$

\section{Cocoa polyphenols and vascular function}

Among healthy individuals, as well as postmenopausal women, the consumption of polyphenols present in cocoa has been associated with a dose-dependent improvement of vascular function, in particular of arterial stiffness measured by pulse wave speed. ${ }^{1314} 19$ One of these studies also suggests that the reduction in arterial stiffness observed in postmenopausal women after consumption of cocoa is independent of the frequency of the intake. ${ }^{19}$ However, this relationship is not evident in people with mild hypertension when cardio-ankle vascular index (CAVI) is used as a measure of arterial stiffness. ${ }^{20}$

There is also evidence of the influence of these polyphenols in reducing the augmentation index (AIx). The study by West $e t a l,{ }^{21}$ involving subjects with excess weight and moderate obesity, concludes that treatment with dark chocolate decreases AIx in women, although it seems that this association might have a greater effect on the elasticity of the large arteries, especially in subjects with obesity and diabetes mellitus type $2 .^{22}$

\section{Cocoa polyphenols and cognitive performance}

There is evidence to suggest that chocolate rich in polyphenols is beneficial for cognitive performance and state since it improves mental processing speed and attenuates the increase of mental fatigue among healthy young adults. ${ }^{23} 24$ An improvement in cognitive performance among older age groups after eating chocolate has also been observed, ${ }^{25}$ especially in subjects with higher risk of cardiovascular disease. ${ }^{26}$ Several studies also show that polyphenol-rich chocolate causes an improvement in executive function, categorical fluency ${ }^{27}$ and working memory, ${ }^{28} 29$ and a slowing of mental fatigue. ${ }^{30}$ Also, a higher frequency of chocolate consumption has been associated with improved cognitive function. ${ }^{29}$ Furthermore, a positive influence of cocoa polyphenols on physiological processes has been reported, with a neuroprotective effect ${ }^{31}$ and improved cognitive performance. ${ }^{32}$ In this regard, it has been suggested that the brain-derived neurotrophic factor plays a role in the cognitive enhancement induced by the flavonoides. ${ }^{33}$ Favourable effects on cerebrovascular function have also been observed in postmenopausal women after consumption of chocolate with a high concentration of cocoa. ${ }^{34}$

\section{Cocoa polyphenols and quality of life}

The quality of life linked to health is represented by the individual's perception of well-being in various aspects of life, including physical and mental aspects. The effect of chocolate and polyphenols on the quality of life has scarcely been studied, with little available evidence and even less of a conclusive nature. In a study conducted among healthy people, where regular consumption of chocolate was recorded over 1 year, no evidence was found of a clear association between chocolate intake and the physical or mental components of quality of life. ${ }^{35}$ Nevertheless, it has been observed that the consumption of dark chocolate might be beneficial for the quality of life of women with fibromyalgia. ${ }^{36}$

\section{Cocoa polyphenols and body composition}

The menopause period leads to various changes in the body composition of women. ${ }^{37}$ Regarding the connection between cocoa polyphenols and body composition, results diverge. Some clinical trials involving healthy people and overweight or obese patients have not reported significant differences that link chocolate consumption to anthropometric measures. ${ }^{16202138}$ Other studies indicate that chocolate consumption might have positive effects on body composition in adolescents, ${ }^{39}$ patients with diabetes ${ }^{40}$ or women with obesity. ${ }^{41}$ Two recent systematic reviews also indicate that eating chocolate is associated with reduced body mass index (BMI) and waist circumference, ${ }^{42} 43$ and one of them also concludes that the amount and the length of time during which it is eaten play a key role in these beneficial effects. ${ }^{43}$ Conversely, other studies such as that carried out with the cohort of the Atherosclerosis Risk in Communities study have observed a dose-dependent increase in weight after habitual chocolate consumption. ${ }^{44}$

In sum, the polyphenols present in chocolate seem to have a positive effect on blood pressure, vascular function, cognitive performance and quality of life, especially in populations with increased cardiovascular risk, such as postmenopausal women. ${ }^{45}$ However, the conflicting results obtained in different studies suggest that the real contribution of these compounds to health and the underlying mechanisms remain unclear. Moreover, most of these studies have used preparations with high concentrations of polyphenols that are usually not present in the normal diet.

This study aims to evaluate the effect of adding a daily amount of $10 \mathrm{~g}$ of chocolate high in cocoa content $(99 \%)$ 
and polyphenols to the normal diet on blood pressure, vascular function, cognitive performance, quality of life and body composition in postmenopausal women.

\section{METHODS AND ANALYSIS Design and setting}

This controlled and randomised clinical trial involves two parallel groups. The study will be carried out in the Research Unit of the La Alamedilla Health Centre in Salamanca (Spain), which is part of the Biomedical Research Institute of Salamanca and the Primary Care Prevention and Health Promotion Research Network. The recruitment schedule is set to start on 1 June 2018, and the study will run until 31 May 2019. There will be a baseline assessment and two follow-ups, at 3 and 6 months.

\section{Study population}

Those subjects who meet the selection criteria and sign the informed consent after receiving information about the objectives and implementation of the study will take part.

Inclusion criteria: women between 50 and 64 years in postmenopause, defined by and checked against amenorrhoea during at least 12 consecutive months.

Exclusion criteria: a personal history of cardiovascular disease; personal history of diabetes mellitus, arterial hypertension or dyslipidaemia under pharmacological treatment; hypocaloric diets; clinically demonstrable neurological and/or neuropsychological disease; treatment with hormone replacement therapy; intolerance and/or allergy to cocoa or any of the components of the supplement.

Participants will be selected using a consecutive sample of women who meet the selection criteria in the general practitioner surgeries of four urban primary care centres in Salamanca, from 1 June, 2018.

\section{Patient and public involvement}

Patients and the public were not involved in the design of this study or outcome measures. We hope that the results of the study will be disseminated through press releases and information-sharing meetings with the study participants.

\section{Sample size}

The size of the sample has been estimated based on the potential modification of the main variable, systolic blood pressure (SBP). Given alpha and beta risks of 0.05 and 0.20 respectively in bilateral contrast and an SD of $5.8 \mathrm{~mm} \mathrm{Hg}, 140$ participants (70 per group) will be necessary to detect a minimum difference of $2.9 \mathrm{~mm} \mathrm{Hg}$ in the SBP between the two groups. A predicted drop-out rate of $10 \%$ during follow-up has been taken into account. This estimate has considered the results obtained in a similar study in which a decrease in SBP of 6.5 was observed $\pm 5.8 \mathrm{~mm} \mathrm{Hg}$. ${ }^{14}$

\section{Randomisation}

Participants will be assigned to the intervention group (IG) or control group (CG) at random. The allocation sequence will be generated by an independent researcher using the Epidat V.4.2 program ${ }^{46}$ before the inclusion of the first participant, using masked block randomisation. Patients will receive their randomisation number based on the order of their baseline evaluation visit and will remain hidden until the participants are assigned to each group. To ensure that the blinding is maintained, patients will be given clear instructions not to disclose which treatment they have been randomised to while being interviewed by the blind assessors. Information on treatment allocation will be stored in a secure locker in case of emergency unblinding.

\section{Intervention}

No type of intervention will be carried out with the CG participants.

IG participants will be given chocolate with $99 \%$ cocoa content and asked to eat $10 \mathrm{~g}$ daily for 6 months. According to the European Food Safety Authority, $10 \mathrm{~g}$ of high-flavanol dark chocolate consumed in the context of a balanced diet could help maintain endothelium-dependent vasodilation. ${ }^{47}$ Participants will also be given instructions on eating and keeping the product, with the recommendations, for example, that the chocolate can be consumed in small pieces leaving them unmated in the mouth, without chewing them. Also, a series of recommendations will be given addressing the organoleptic characteristics of the product, as well as the recommendations of trying to consume the product at the same time or refrain from ingesting it dissolved in milk. Also, participants will be given a calendar on which to record the time it was eaten each day. This calendar will be returned to the researchers at each replenishment visit.

This amount of chocolate provides the following daily nutritional contribution: $59 \mathrm{kcal}, 0.8 \mathrm{~g}$ of carbohydrates, $1.5 \mathrm{~g}$ of protein, $5.1 \mathrm{~g}$ of fat, of which $3.1 \mathrm{~g}$ are saturated fats. The proportion of polyphenols per $10 \mathrm{~g}$ is $65.4 \mathrm{mg}$. The polyphenolic profile of this compound can be seen in table 1. On each visit, IG participants will receive the amount of chocolate they need until the

\begin{tabular}{ll}
\hline Table 1 Polyphenols composition of 99\% cocoa chocolate \\
\hline Compounds & Quantity \\
\hline Protocatechuic acid (mg/g) & $0.058 \pm 0.008$ \\
\hline Procyanidin dimer (B3) (mg/g) & $0.176 \pm 0.013$ \\
\hline Catechin $(\mathrm{mg} / \mathrm{g})$ & $1.035 \pm 0.105$ \\
\hline Procyanidin dimer (B2) (mg/g) & $1.440 \pm 0.055$ \\
\hline Epicatechin (mg/g) & $2.610 \pm 0.075$ \\
\hline Procyanidin trimer (C1) (mg/g) & $0.853 \pm 0.024$ \\
\hline Procyanidin A hexoside $(\mathrm{mg} / \mathrm{g})$ & $0.354 \pm 0.007$ \\
Quercetin glucoside $(\mathrm{mg} / \mathrm{g})$ & $0.002 \pm 0.000$ \\
Quercetin arabinoside $(\mathrm{mg} / \mathrm{g})$ & $0.003 \pm 0.001$ \\
\hline
\end{tabular}


next replenishment visit. In addition to the baseline visit, there will be five replenishment visits in months 1, 2, 3 (coinciding with the evaluation visit), 4 and 5 . The sole purpose of the replenishment visits will be to supply the amount of chocolate needed until the next visit, without any other intervention being carried out.

Participants in both groups will be instructed to continue with the dietary pattern they usually follow, without changing their eating habits during the study period.

\section{Procedures}

For each participant a baseline visit and two follow-up visits at 3 and 6 months after the initial one are scheduled (figure 1). The IG will also make five replenishment visits, in months 1, 2, 3 (coinciding with the first follow-up visit), 4 and 5 . In the replenishment visits, participants will be given the amount of chocolate needed until the next visit and will hand in the calendar with the record of the chocolate eaten.

\section{Primary and secondary endpoints}

The primary variable will be the decrease in clinical blood pressure, measured with a digital sphygmomanometer. Secondary variables will include vascular function, quality of life, cognitive performance and body composition.

All variables will be measured at 3 and 6 months after randomisation, except for cognitive performance and quality of life, which will be assessed only after 6 months.

\section{Blood pressure}

Clinical systolic and diastolic blood pressure will be measured with a validated Omron M10-IT sphygmomanometer (Omron Healthcare, Kyoto, Japan). Three measurements will be taken in the dominant arm of the subject in a sitting position after at least $5 \mathrm{~min}$ of rest with an appropriately sized cuff, following the recommendations of the European Society of Hypertension. ${ }^{48}$ The average of the last two measurements will be recorded.

\section{Vascular function}

The Vasera VS-2000 device (Fukuda Denshi) will be used to measure the CAVI and the brachial-ankle pulse wave velocity (ba-PWV) at rest. CAVI is a good indicator of arterial stiffness, providing an accurate estimate of the degree of atherosclerosis without depending on blood pressure. ${ }^{49} \mathrm{CAVI} \geq 9$ and ba-PWV $\geq 18.3$ will be considered pathological. ${ }^{50}$ Pathological CAVI is representative of subclinical atherosclerosis. ${ }^{51}$

\section{Cognitive performance}

The instructions are presented visually at the start of the baseline measurement to ensure limiting a learning effect over the subsequent testing periods. Attention and executive functions: Trail Making Test A will be used to measure attention and Trail Making Test B for processing speed and executive functions. ${ }^{52}$
Immediate verbal memory will be assessed with the Rey Auditory Verbal Learning Test. The immediate recall of a list of 15 words is measured in three attempts, followed by delayed verbal memory through the free recall of the words learnt in the first part of the test after $10 \mathrm{~min} .^{53}$

Working memory will be assessed with the WAIS Digit Span Backward test. ${ }^{54}$

Phonological fluency will be explored by naming as many words as possible starting with different letters of the FAS Questionnaire in the space of 1 min. ${ }^{55}$

Categorical fluency measures verbal semantic fluency and will be assessed by naming as many animals as possible in 1 min. ${ }^{56}$

\section{Quality of life}

The quality of life linked to health will be assessed through the EuroQol 5-D questionnaire. We will use the adapted Spanish version of this questionnaire, which has been validated in the Spanish population. ${ }^{57}$ This questionnaire consists of three elements: the assessment by the individuals of their state of health in level of severity by dimension (mobility, personal care, daily activities, pain/discomfort and anxiety/depression), the assessment of their state of health on an analogue visual scale and finally an index of social values obtained for each state of health generated by the instrument.

The quality of life will also be studied using the Cervantes Scale. ${ }^{58}$ This questionnaire is specifically designed for menopause and postmenopause and has been validated for Spanish women. Its 31 structured items cover the four dimensions of menopause: menopause and health, sexuality, psychic domain and relationships.

\section{Body composition}

Body composition will be measured with the Inbody 230 Monitor. ${ }^{59}$ This analyser provides data on fat mass and body fat percentage as principal outcomes and also skeletal muscle mass, total body water, fat-free mass, waist-hip ratio, basal metabolism and a segmental analysis.

Body weight will be measured twice with an electronic scale (Scale 7830, Soehnle Professional, Backnang, Germany) after proper calibration (accuracy $\pm 0.1 \mathrm{~kg}$ ). Height will be measured by recording the average of two readings rounded to the nearest centimetre using a stadiometer (Seca 222, Medical Scale and Measurement System, Birmingham, UK). Both measurements will be made with the subject barefoot and wearing light clothing. BMI will be calculated by dividing weight $(\mathrm{kg})$ by height squared $\left(\mathrm{m}^{2}\right)$. Waist circumference will be assessed in accordance with the recommendations of the Spanish Society for the Study of Obesity ${ }^{60}$ and will be measured in duplicate before and after inhalation, using a flexible tape parallel to the floor, at the level of the midpoint between the lowest rib and the iliac crest, with the subject standing up and without clothes. 


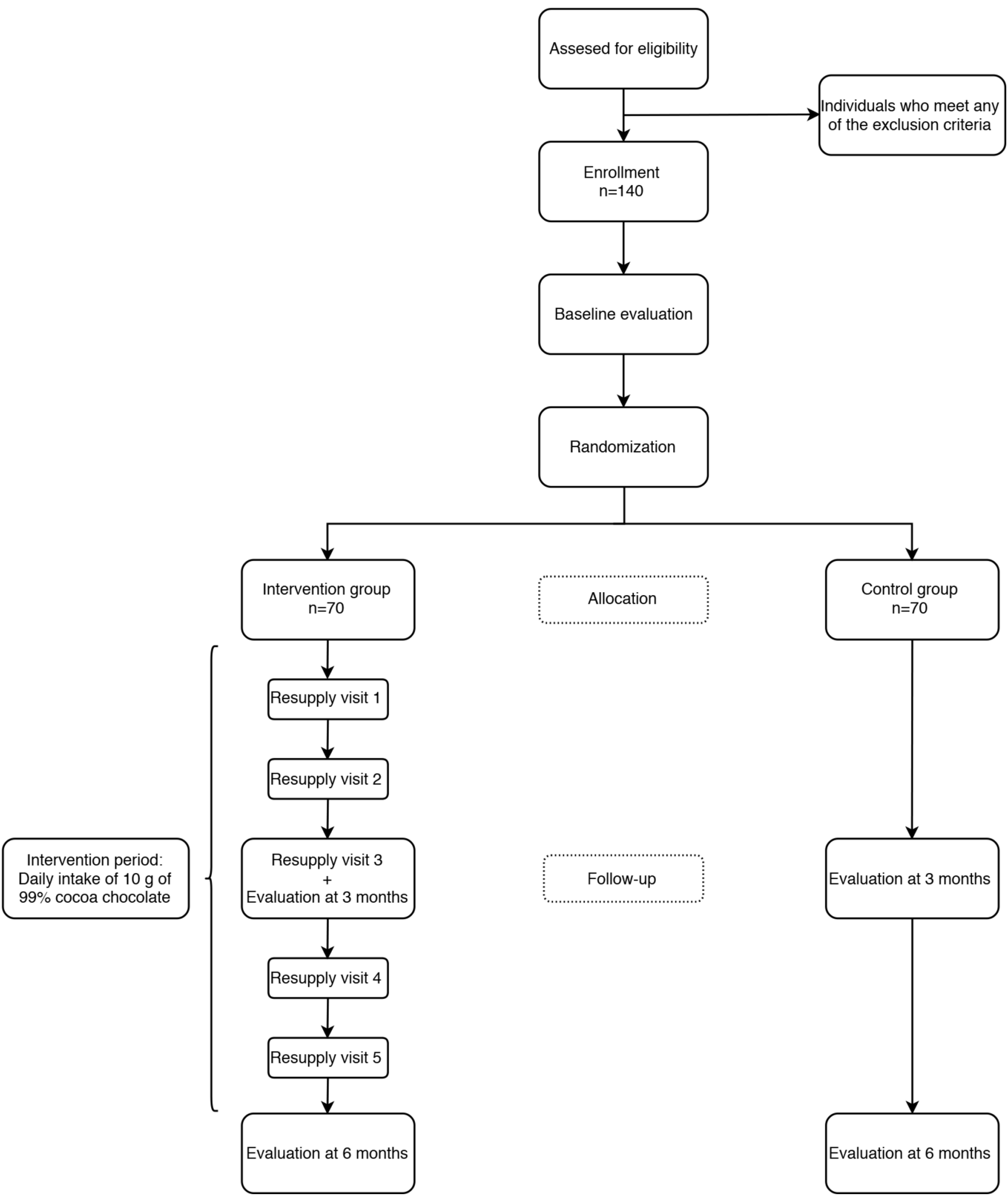

Figure 1 Study flow chart.

\section{Other variables}

Clinical and sociodemographic variables

At the baseline visit, information on clinical and sociodemographic variables will also be collected via questions about age, marital status, educational level and occupation. The family history of cardiovascular disease and personal history of anxiety and depression, gestational diabetes, hypertension, dyslipidaemia and the prescribed pharmacological treatment (antiaggregants, anticoagulants, thyroid hormone treatment, anxiolytics) 
will also be recorded, as well as the taking of non-steroidal anti-inflammatory drugs in the last two weeks.

In subsequent visits, personal histories of cardiovascular disease, diabetes mellitus, arterial hypertension or dyslipidaemia in treatment, as well as the prescribed pharmacological treatment (hypolipidaemic, antihypertensive, antidiabetic) will also be noted.

\section{Evaluation of chocolate consumption and habitual diet}

Chocolate consumption will be assessed at each evaluation visit by a series of questions about the amount, type and frequency of consumption in the period between visits.

Nutritional habits will be assessed by a 24 hours log on three non-consecutive days prior to each visit.

\section{Evaluation of other lifestyles}

The use of tobacco will be assessed with a questionnaire on the personal history and pattern of smoking.

Alcohol use will be recorded with a questionnaire covering the previous seven days, which will include specific beverages and the amount by volume drunk of each.

Physical activity will be measured using the International Physical Activity Questionnaire in its short version and validated in Spanish. ${ }^{61}$ This questionnaire measures activity over the previous seven days, classifying the subjects according to three activity levels (low, moderate and high) with respect to three types of activities: walking, moderate-intensity activities and vigorous-intensity activities. The amount of physical exercise will be estimated in METs-minute/week.

\section{Evaluation of laboratory variables}

At baseline and follow-up visits at 6 months, we will measure plasma fasting glucose values $(\mathrm{mg} / \mathrm{dL})$, glycated haemoglobin $(\%)$, total cholesterol $(\mathrm{mg} / \mathrm{dL})$, total triglycerides $(\mathrm{mg} / \mathrm{dL})$, high-density lipoprotein cholesterol $(\mathrm{mg} / \mathrm{dL})$, low-density lipoprotein cholesterol $(\mathrm{mg} / \mathrm{dL})$, creatinine $(\mathrm{mg} / \mathrm{L})$ and insulinaemia $(\mathrm{mg} / \mathrm{dL})$. Creatinine in urine $(\mathrm{mg} / \mathrm{dL})$ and microalbuminuria $(\mathrm{mg} / \mathrm{dL})$ will also be measured. Insulin resistance will be determined using the Homeostasis Model Assessment Insulin Resistance index estimated using the following equation: Fasting glucose $(\mathrm{mmol} / \mathrm{L}) \times$ insulin $(\mathrm{mU} / \mathrm{mL}) / 22.5$.

The evaluation visits will be made in the morning between 08:00 and 10:00. Each participant will be informed prior to the visit to fast for at least 12 hours, having avoided during the 24 hours prior to visiting the consumption of polyphenol-rich foods, including cocoa, chocolate, apples and red wine as well as alcoholic drinks or the performance of the programmed physical activity. All evaluation visits, including blood pressure measurements and evaluations of vascular function, will be carried out in a room with standardised lighting and temperature, recommending that patients attend the appointment with a prior rest of at least 8-10 hours.
Data collection procedure, data management and monitoring

Data collection of the baseline and follow-up evaluation visits at 3 and 6 months will be carried out by a nurse specifically trained to do so. The intervention visit after the baseline evaluation will be carried out by another nurse, different from the one who performs the data collection. Each participant will have a unique identification code within the study. All measurements will be compiled in a data collection notebook and kept in a secure place that will remain closed within the health centre. A database will be created in SPSS to which only the members of the research team and the people related to the statistical analyses will have access. The principal investigator or a person designated for this purpose will perform a weekly process of monitoring the study, taking into account the inclusion of patients, cleaning and debugging of databases, and adaptation of the procedures to the protocol.

\section{Blinding strategy}

Due to the nature of the intervention itself, the participants and the person responsible for delivering the chocolate to IG participants cannot be blinded. However, the person responsible for carrying out the study measurements at each visit and for the statistical analysis will be blind to the intervention.

\section{Statistical analysis}

General analysis

Results for the quantitative variables will be expressed by mean $\pm \mathrm{SD}$ or by frequency distribution in the case of qualitative variables. The normality of the variables will be assessed using the Kolmogorov-Smirnov test. In cases where a normal distribution cannot be assumed, the corresponding non-parametric tests will be applied. The association between independent qualitative variables will be analysed using the $\chi^{2}$ test or Fisher's exact test. The means between the two groups will be compared using the Student's t-test or the Mann-Whitney U test, and the Pearson or Spearman correlation coefficients will be calculated to analyse the relationship between quantitative variables.

The analysis of the results for the main variable and the secondary variables will be carried out by intention to treat. Also, a secondary analysis will be made, taking into account chocolate intake adherence $(<50 \%$ days and $>50 \%$ days) and other relevant subgroups in relation to their physical activity or previous chocolate consumption.

All analyses will be performed using SPSS V.23.0 (IBM) and an alpha risk of 0.05 will be set as the limit of statistical significance.

\section{Analysis of the intervention's effect on primary and secondary} outcomes

To analyse the changes at 3 and 6 months from baseline in the primary outcome (blood pressure) and in the secondary outcomes within the same group, the Student's t-test for paired data or the Wilcoxon test will be used. 
The McNemar test will be applied with quantitative or dichotomous variables.

Effects of the intervention will be analysed in a comparison of the changes in blood pressure and the secondary variables between the IG and the CG using analysis of covariance and adjusting for possible confounders, for example, smoking status. Effects of the intervention during follow-up will be studied with an analysis of the variance of repeated measures.

\section{Analysis by subgroups}

The effect of the intervention could be influenced by age, sociocultural level and adherence to the study's chocolate intake. The same analyses described above will be performed for each of the subgroups above.

\section{Secondary analyses}

A multivariate multiple regression analysis will be performed to identify the variables with the greatest influence on blood pressure changes and the secondary variables analysed.

\section{Methodological limitations}

Due to the nature of the intervention, the participating subjects cannot be blinded. However, the researcher who analyses the data and the person who makes the measurements during follow-up visits will be blinded with respect to the group to which the participants belong. The smoking status in the 12 months prior to the time of inclusion could influence the outcome measures related to vascular function and blood pressure; therefore, although these participants will not be excluded, this aspect will be controlled in the statistical analysis. Assessment of the quality of life and lifestyles will be carried out through self-reported data; however, previously validated instruments will be used to obtain these. To make compliance with the intervention in the IG easier, IG participants will be provided with instructions on eating the chocolate and a calendar to record each intake.

\section{ETHICS AND DISSEMINATION \\ Ethical considerations}

The study was approved by the Clinical Research Ethics Committee of the Salamanca Health Area ('CREC of Health Area of Salamanca') in February 2018. A SPIRIT checklist is available for this protocol. The clinical trial has been registered at ClinicalTrials.gov with the identifier NCT03492983.

Participants must provide informed consent in accordance with the Declaration of Helsinki. Subjects will be informed of the objectives of the project and the risks and benefits of the explorations to be carried out, including sample collection. None of the tests will pose risks that could endanger the lives of participants. Confidentiality of participant data will be guaranteed at all times in accordance with the provisions of the Organic Law on the Protection of Personal Data (15/1999 of 13 December
LOPD), and under the conditions established by Law $14 / 2007$ of biomedical research.

\section{Dissemination plan}

The research group plans to achieve rapid and widespread dissemination of results to ensure maximum visibility of this study. To this end, the results of the study will be published in open-access scientific journals with peer review. At least one publication of the main results and others with the secondary results are planned. This will be complemented by the presentation of the results of the study at relevant scientific conferences and seminars of national and international scope. Also, a doctoral thesis based on this project will be prepared. Appropriate dissemination will likewise be carried out through social networks and other media. Moreover, given the involvement of a commercial product, the transfer to clinical practice is expected to be rapid if the results are as expected.

\section{DISCUSSION}

In recent years, there has been an increase in attention to polyphenols and their beneficial effects on health, with numerous studies being carried out to assess this. ${ }^{192}$ Similarly, the therapeutic use of these compounds has been suggested for certain diseases or population groups. ${ }^{36} 62$ The menopause increases the risk of developing cardiovascular disease compared with the previous period. ${ }^{45}$ However, we have not found any study that assesses the effect of adding commercially available chocolate high in cocoa content to the usual diet in this population. Similarly, no studies have been found that evaluate the effects on cognitive performance, quality of life and body composition of adding commercial chocolate with high cocoa content to the usual diet in postmenopausal women.

This work will provide novel data helpful for the development of strategies in the nutritional education of particularly vulnerable populations, given their high risk of developing cardiovascular disease, including non-pharmacological therapies and strategies that employ lifestyle modification. This intervention might also have implications for the preparation of recommendations in clinical practice guidelines and quality improvement programmes aimed at the care of postmenopausal women.

\section{Author affiliations \\ ${ }^{1}$ Primary Health Care Research Unit, La Alamedilla Health Center, Institute of Biomedical Research of Salamanca (IBSAL), Health Service of Castilla y León (SACyL), Salamanca, Spain \\ ${ }^{2}$ Gerencia de Atención Primaria de Burgos, SACYL, Burgos \\ ${ }^{3}$ Department of Biomedical and Diagnostic Sciences, University of Salamanca, \\ Salamanca, Spain \\ ${ }^{4}$ Department of Medicine, University of Salamanca, Salamanca, Spain \\ ${ }^{5}$ Department of Nursing, University of Extremadura, Plasencia, Spain \\ ${ }^{6}$ Department of Basic Psychology, Psychobiology and Behavioral Sciences Methodology, University of Salamanca, Salamanca, Spain \\ ${ }^{7}$ Department of Analytical Chemistry, University of Salamanca, Salamanca, Spain \\ ${ }^{8}$ Faculty of Health Sciences, University of Burgos, Burgos, Spain}


Acknowledgements The authors are grateful to all the professionals involved in the ECCAMP study: José I Recio-Rodríguez, José A Maderuelo-Fernández, Luis García-Ortiz, Manuel A Gómez-Marcos, Irene A García-Yu, Rosario Alonso-Domínguez, Sara Mora-Simón, Natalia Sánchez-Aguadero, Jesús González-Sánchez, Cristina Agudo-Conde, Cristina Lugones-Sánchez, Benigna Sánchez-Salgado, Carmen Castaño-Sánchez, Emiliano Rodríguez-Sánchez, Susana González-Manzano, Olaya Tamayo-Morales, and Susana González-Sánchez.

Contributors JI-RR, JAM-F, LG- 0 and IAG-Y contributed to the conception and design of the study. IAG-Y, JIR-R and JAM-F prepared the manuscript of the study protocol. JIR-R, JAM-F, LG-0, RA-D, SM-S, JG-S, SM-G, ER-S, MAG-M and IAG-Y contributed to the development of the study protocol. JI-RR, JAM-F, LG-0, RA-D, SMS, JGS, SMG, ERS, MGM and IAG-Y provided assistance with statistical methodology and knowledge. JI-RR, JAM-F, LG-0, RA-D, SM-S, JG-S, SM-G, ER-S, MG-M and IAG-Y provided a critical review of the manuscript. All authors have read and accepted the final version of the protocol.

Funding This study was supported in part by grants funded by la Gerencia Regional de Castilla y León (GRS 1583/B/17).

Disclaimer Lindt \& Sprüngli will provide the necessary chocolate for the implementation of the study. This company will not play any role in the design of the study, data analysis, reporting of results or the decision to present the manuscript for publication.

Competing interests None declared.

Patient consent for publication Obtained.

Ethics approval Clinical Research Ethics Committee of the Salamanca Health Area ("CREC of Health Area of Salamanca").

Provenance and peer review Not commissioned; externally peer reviewed.

Open access This is an open access article distributed in accordance with the Creative Commons Attribution Non Commercial (CC BY-NC 4.0) license, which permits others to distribute, remix, adapt, build upon this work non-commercially, and license their derivative works on different terms, provided the original work is properly cited, appropriate credit is given, any changes made indicated, and the use is non-commercial. See: http://creativecommons.org/licenses/by-nc/4.0/.

\section{REFERENCES}

1. Grassi D, Desideri G, Ferri C. Flavonoids: antioxidants against atherosclerosis. Nutrients 2010;2:889-902.

2. Grassi D, Desideri G, Croce G, et al. Flavonoids, vascular function and cardiovascular protection. Curr Pharm Des 2009;15:1072-84.

3. Visioli F, Bernaert H, Corti R, et al. Chocolate, lifestyle, and health. Crit Rev Food Sci Nutr 2009;49:299-312.

4. Cos P, De Bruyne T, Hermans N, et al. Proanthocyanidins in health care: current and new trends. Curr Med Chem 2004;11:1345-59.

5. Grassi D, Desideri G, Ferri C. Blood pressure and cardiovascular risk: what about cocoa and chocolate? Arch Biochem Biophys 2010;501:112-5.

6. Mink PJ, Scrafford CG, Barraj LM, et al. Flavonoid intake and cardiovascular disease mortality: a prospective study in postmenopausal women. Am J Clin Nutr 2007;85:895-909.

7. Ramos S, Martín MA, Goya L. Effects of cocoa antioxidants in type 2 diabetes mellitus. Antioxidants 2017:6:84.

8. Osakabe N. Flavan 3-ols improve metabolic syndrome risk factors: evidence and mechanisms. J Clin Biochem Nutr 2013;52:186-92.

9. Engler MB, Engler MM, Chen CY, et al. Flavonoid-rich dark chocolate improves endothelial function and increases plasma epicatechin concentrations in healthy adults. J Am Coll Nutr 2004;23:197-204.

10. Ottaviani JI, Heiss C, Spencer JPE, et al. Recommending flavanols and procyanidins for cardiovascular health: Revisited. Mol Aspects Med 2018;61:63-75.

11. Ludovici V, Barthelmes J, Nägele MP, et al. Cocoa, blood pressure, and vascular function. Front Nutr 2017;4:36

12. Ried K, Fakler P, Stocks NP. Effect of cocoa on blood pressure. Cochrane Database Syst Rev 2017;4:CD008893.

13. Heiss C, Sansone R, Karimi H, et al. FLAVIOLA Consortium, European Union 7th Framework Program. Impact of cocoa flavanol intake on age-dependent vascular stiffness in healthy men: a randomized, controlled, double-masked trial. Age 2015;37:9794.

14. Grassi D, Desideri G, Necozione S, et al. Cocoa consumption dosedependently improves flow-mediated dilation and arterial stiffness decreasing blood pressure in healthy individuals. J Hypertens 2015;33:294-303.
15. Crews WD, Harrison DW, Wright JW. A double-blind, placebocontrolled, randomized trial of the effects of dark chocolate and cocoa on variables associated with neuropsychological functioning and cardiovascular health: clinical findings from a sample of healthy, cognitively intact older adults. Am J Clin Nutr 2008;87:872-80.

16. Dower JI, Geleijnse JM, Gijsbers L, et al. Effects of the pure flavonoids epicatechin and quercetin on vascular function and cardiometabolic health: a randomized, double-blind, placebocontrolled, crossover trial. Am J Clin Nutr 2015;101:914-21.

17. Modena MG. Hypertension in postmenopausal women: how to approach hypertension in menopause. High Blood Press Cardiovasc Prev 2014;21:201-4.

18. Zilberman JM, Cerezo GH, Del Sueldo M, et al. Association between hypertension, menopause, and cognition in women. J Clin Hypertens 2015;17:970-6.

19. Okamoto T, Kobayashi R, Natsume M, et al. Habitual cocoa intake reduces arterial stiffness in postmenopausal women regardless of intake frequency: a randomized parallel-group study. Clin Interv Aging 2016;11:1645-52.

20. Koli R, Köhler K, Tonteri E, et al. Dark chocolate and reduced snack consumption in mildly hypertensive adults: an intervention study. Nutr J 2015;14:84.

21. West SG, Mclntyre MD, Piotrowski MJ, et al. Effects of dark chocolate and cocoa consumption on endothelial function and arterial stiffness in overweight adults. Br J Nutr 2014;111:653-61.

22. Basu A, Betts NM, Leyva MJ, et al. Acute Cocoa Supplementation Increases Postprandial HDL Cholesterol and Insulin in Obese Adults with Type 2 Diabetes after Consumption of a High-Fat Breakfast. J Nutr 2015;145:2325-32.

23. Scholey AB, French SJ, Morris PJ, et al. Consumption of cocoa flavanols results in acute improvements in mood and cognitive performance during sustained mental effort. J Psychopharmacol 2010;24:1505-14.

24. Field DT, Williams CM, Butler LT. Consumption of cocoa flavanols results in an acute improvement in visual and cognitive functions. Physiol Behav 2011;103:255-60.

25. Sorond FA, Hurwitz S, Salat DH, et al. Neurovascular coupling, cerebral white matter integrity, and response to cocoa in older people. Neurology 2013;81:904-9.

26. Socci V, Tempesta D, Desideri G, et al. Enhancing Human Cognition with Cocoa Flavonoids. Front Nutr 2017;4:19.

27. Mastroiacovo D, Kwik-Uribe C, Grassi D, et al. Cocoa flavanol consumption improves cognitive function, blood pressure control, and metabolic profile in elderly subjects: the Cocoa, Cognition, and Aging (CoCoA) Study--a randomized controlled trial. Am J Clin Nutr 2015;101:538-48.

28. Grassi D, Socci V, Tempesta D, et al. Flavanol-rich chocolate acutely improves arterial function and working memory performance counteracting the effects of sleep deprivation in healthy individuals. $J$ Hypertens 2016;34:1298-308.

29. Crichton GE, Elias MF, Alkerwi A. Chocolate intake is associated with better cognitive function: The Maine-Syracuse Longitudinal Study. Appetite 2016;100:126-32.

30. Massee LA, Ried K, Pase M, et al. The acute and sub-chronic effects of cocoa flavanols on mood, cognitive and cardiovascular health in young healthy adults: a randomized, controlled trial. Front Pharmacol 2015;6:93.

31. Nehlig A. The neuroprotective effects of cocoa flavanol and its influence on cognitive performance. $\mathrm{Br} J \mathrm{Clin}$ Pharmacol 2013;75:716-27.

32. Nurk E, Refsum H, Drevon $\mathrm{CA}$, et al. Intake of flavonoid-rich wine, tea, and chocolate by elderly men and women is associated with better cognitive test performance. J Nutr 2009;139:120-7.

33. Neshatdoust S, Saunders C, Castle SM, et al. High-flavonoid intake induces cognitive improvements linked to changes in serum brainderived neurotrophic factor: Two randomised, controlled trials. Nutr Healthy Aging 2016;4:81-93.

34. Marsh CE, Carter HH, Guelfi KJ, et al. Brachial and Cerebrovascular Functions Are Enhanced in Postmenopausal Women after Ingestion of Chocolate with a High Concentration of Cocoa. J Nutr 2017;147:jn250225-92.

35. Balboa-Castillo T, López-García E, León-Muñoz LM, et al. Chocolate and health-related quality of life: a prospective study. PLoS One 2015;10:e0123161

36. Costa de Miranda R, Paiva ES, Suter Correia Cadena SM, et al. Polyphenol-rich foods alleviate pain and ameliorate quality of life in fibromyalgic women. Int J Vitam Nutr Res 2016:1-10.

37. Dmitruk A, Czeczelewski J, Czeczelewska E, et al. Body composition and fatty tissue distribution in women with various menstrual status. Rocz Panstw Zakl Hig 2018;69:95-101. 
38. Davison K, Coates AM, Buckley JD, et al. Effect of cocoa flavanols and exercise on cardiometabolic risk factors in overweight and obese subjects. Int J Obes 2008;32:1289-96.

39. Cuenca-García M, Ruiz JR, Ortega FB, et al. HELENA study group. Association between chocolate consumption and fatness in European adolescents. Nutrition 2014;30:236-9.

40. Ayoobi N, Jafarirad S, Haghighizadeh $\mathrm{MH}$, et al. Protective effect of dark chocolate on cardiovascular disease factors and body composition in type 2 diabetes: a parallel, randomized, clinical trial. Iran Red Crescent Med J 2017;19:e21644.

41. Di Renzo L, Rizzo M, Sarlo F, et al. Effects of dark chocolate in a population of normal weight obese women: a pilot study. Eur Rev Med Pharmacol Sci 2013;17:2257-66.

42. González-Sarrías A, Combet E, Pinto P, et al. A systematic review and meta-analysis of the effects of flavanol-containing tea, cocoa and apple products on body composition and blood lipids: exploring the factors responsible for variability in their efficacy. Nutrients $2017 ; 9: 746$

43. Kord-Varkaneh H, Ghaedi E, Nazary-Vanani A, et al. Does cocoa/dark chocolate supplementation have favorable effect on body weight, body mass index and waist circumference? A systematic review, meta-analysis and dose-response of randomized clinical trials. Crit Rev Food Sci Nutr 2018;0:1-14.

44. Greenberg JA, Buijsse B. Habitual chocolate consumption may increase body weight in a dose-response manner. PLOS One 2013;8:e70271.

45. Agrinier N, Cournot M, Dallongeville J, et al. Menopause and modifiable coronary heart disease risk factors: a population based study. Maturitas 2010;65:237-43.

46. Consellería de Sanidade Xunta de Galicia. Spain; Pan American Organization health (PAHO-WHO); CES University C. Epidat: program for epidemiological data analysis. Version 4.2Julio. 2016.

47. Scientific Opinion on the substantiation of a health claim related to cocoa flavanols and maintenance of normal endothelium-dependent vasodilation pursuant to Article 13(5) of Regulation (EC) No 1924/2006. EFSA Journal 2012;102809.

48. Allen JK, Stephens J, Dennison Himmelfarb CR, et al. Randomized controlled pilot study testing use of smartphone technology for obesity treatment. J Obes 2013;2013:1-7.
49. Shirai K, Hiruta N, Song M, et al. Cardio-ankle vascular index (CAVI) as a novel indicator of arterial stiffness: theory, evidence and perspectives. J Atheroscler Thromb 2011;18:924-38.

50. Ohkuma T, Tomiyama H, Ninomiya T, et al. Proposed Cutoff Value of Brachial-Ankle Pulse Wave Velocity for the Management of Hypertension. Circ J 2017;81:1540-2.

51. Korkmaz L, Erkan H, Korkmaz AA, et al. Relationship of aortic knob width with cardio-ankle vascular stiffness index and its value in diagnosis of subclinical atherosclerosis in hypertensive patients: a study on diagnostic accuracy. Anadolu Kardiyol Derg 2012;12:102-6.

52. Reitan RM. Trail making test. Tucson: Reitan Neuropsychology Laboratory, 1992.

53. Rey A. L'examen clinique en psychologie. Paris: Presses universitaires de France, 1964.

54. Wechsler D. WMS-R Wechsler memory scale. San Antonio, Texas: The Psychological Corporation, 1987.

55. Valencia NJ, Laserna JA, Pérez-García M, et al. Influencia de la escolaridad y el sexo sobre la ejecución en el FAS, nombrar animales y nombrar frutas. Psicología Conductual 2000;8:283-95.

56. Goodglass HKE. Evaluación de la Afasia y de Trastornos Relacionados, 1986.

57. Badia X, Schiaffino A, Alonso J, et al. Using the EuroQol 5-D in the Catalan general population: feasibility and construct validity. Qual Life Res 1998;7:311-22.

58. Palacios S, Ferrer-Barriendos J, Parrilla JJ, et al. [Health-related quality of life in the Spanish women through and beyond menopause. Development and validation of the Cervantes Scale]. Med Clin 2004;122:205-11.

59. Karelis AD, Chamberland G, Aubertin-Leheudre M, et al. Validation of a portable bioelectrical impedance analyzer for the assessment of body composition. Appl Physiol Nutr Metab 2013;38:27-32.

60. Salas-Salvadó J, Rubio MA, Barbany M, et al. [SEEDO 2007 Consensus for the evaluation of overweight and obesity and the establishment of therapeutic intervention criteria]. Med Clinquiz $1 p$ following 2007;128:184-96.

61. Román Viñas B, Ribas Barba L, Ngo J, et al. [Validity of the international physical activity questionnaire in the Catalan population (Spain)]. Gac Sanit 2013;27:254-7.

62. Islam MA, Alam F, Solayman M, et al. Dietary phytochemicals: natural swords combating inflammation and oxidation-mediated degenerative diseases. Oxid Med Cell Longev 2016;2016:1-25. 